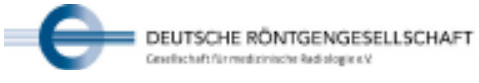

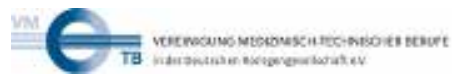

Stellungnahme

\section{Gemeinsam Interessen der MTRA vertreten}

In einem offenen Brief an die Deutsche Röntgengesellschaft haben MTRA Missstände innerhalb ihres Berufs benannt und Verbesserungen eingefordert.

Die Deutsche Röntgengesellschaft e.V., vertreten durch die Vereinigung Medizinisch-Technischer Berufe (VMTB) versteht sich als Unterstützer und Förderer der MTRA in Deutschland. Ihre Aufgabe besteht darin, eine effiziente und fachlich qualifizierte Aus-, Fort- und Weiterbildung zu ermöglichen, die dem stetigen Wandel des Berufsbildes der MTRA Rechnung trägt. Darüber hinaus bemüht sich die VMTB im Rahmen ihrer Möglichkeiten, die Belange der MTRA öffentlich zu machen und notwendige Verbesserungen anzustoßen.

Der offene Brief an die Deutsche Röntgengesellschaft spricht im Wesentlichen 4 Punkte an: Berufsstandwahrung, Ausbildung, Vergütung und Strahlenschutz.

Einen Berufsstandschutz gibt es bereits. MTRA dürfen gemäß MTA-Gesetz selbstständig und eigenverantwortlich arbeiten und Aufnahmen anfertigen. Für bestimmte Tätigkeiten - z.B. im Rahmen der Teleradiologie - müssen MTRA anwesend sein. Die Entscheidung, medizinischem Personal die Möglichkeit zu geben, nach einem erfolgreich absolvierten Strahlenschutzkurs Röntgenaufnahmen unter Aufsicht eines fachkundigen Arzts durchzuführen, war eine politische und können wir leider nicht rückgängig machen. Die Einstellung von MFA mit Röntgenschein ist sicherlich wirtschaftlich motiviert, bewegt sich aber eindeutig innerhalb der gültigen gesetzlichen Regelungen. Problematisch erscheinen hier vielmehr fehlende Kontrollmechanismen, die eine Einhaltung der gesetzlichen Vorgaben gewährleisten. Die Situation auf dem Arbeitsmarkt für MTRA lässt derzeit auch die Berücksichtigung der dienstlichen Rahmenbedingungen bei der Auswahl für eine Anstellung zu.

Rechtliche Grundlage für die Ausbildung von MTRA ist die Ausbildungs- und Prüfungsverordnung für technische Assistenten in der Medizin von 1994. An der Aktualisierung dieser Verordnung arbeiten
DVTA und VMTB schon seit Jahren. Dessen ungeachtet gibt es bei Dozenten - wie auch bei Schülern - sicherlich voneinander abweichende Qualitäten. Die Akademisierung des MTRA-Berufs wird schon seit geraumer Zeit intensiv diskutiert. Auch hier stehen rechtliche und politische Klippen im Weg, die erst noch umschifft werden müssen. Jedoch gibt es bereits jetzt für MTRA mit dem Wunsch nach weitergehender Qualifizierung entsprechende Bachelor-Studiengänge.

Die Forderung nach einer einheitlichen und leistungsgerechten Vergütung ist nachvollziehbar, muss aber an die für MTRA zuständige Vereinte Dienstleistungsgewerkschaft ver.di adressiert werden. Von deren Verhandlungsgeschick hängt es ab, ob sich für MTRA diesbezügliche Verbesserungen ergeben.
Strahlenschutz ist in der Radiologie von herausragender Bedeutung. Ein wichtiger Aspekt ist hier die rechtfertigende Indikation, die ausschließlich vom Radiologen oder einem Arzt mit entsprechender Fachkunde gestellt wird. Eine Röntgenanforderung alleine reicht für eine Bildgebung nicht aus. Für offensichtliche Missstände und Verstöße sind die Gewerbeaufsichtsämter zuständig und sollten im Bedarfsfall kontaktiert werden.

Die VMTB ist bestrebt, MTRA in der Ausübung ihres Berufs bestmöglich zu unterstützen und deren Bedeutung für eine qualitativ hochwertige medizinische Versorgung öffentlich noch bekannter zu machen. Die in dem offenen Brief formulierten Ziele gehen allerdings über die Zuständigkeit und auch Möglichkeiten der VMTB als einer wissenschaftlich geleiteten Interessensgemeinschaft hinaus. Erfolg ist nur gemeinsam möglich - im Interesse aller MTRA in Deutschland. 